\title{
The Representative Curve of Type-2 Fuzzy Data Point Modeling
}

\author{
Rozaimi Zakaria $^{1}$, Abd. Fatah Wahab ${ }^{1} \&$ R. U. Gobithaasan ${ }^{1}$ \\ ${ }^{1}$ Department of Mathematics, Faculty of Science and Technology, Universiti Malaysia Terengganu (UMT), \\ Terengganu, Malaysia \\ Correspondence: Rozaimi Zakaria, Department of Mathematics, Faculty of Science and Technology, Universiti \\ Malaysia Terengganu (UMT), Terengganu 21030, Malaysia. E-mail: rozaimi_z@yahoo.com
}

Received: January 25, 2013

Accepted: March 15, 2013 Online Published: April 19, 2013

doi:10.5539/mas.v7n5p60

URL: http://dx.doi.org/10.5539/mas.v7n5p60

\begin{abstract}
This paper discusses about the construction of type-2 fuzzy data points (T2FDPs) to capture the ambiguity of complex uncertainty data points based on the type-2 fuzzy set theory (T2FST). The construction is based on the type-2 fuzzy number's (T2FN) definition since we deal with the problem of defining the complex uncertainty data points. In order to develop T2FDPs, we use interpolating cubic Bezier curve model for better undestandingof the resultant curve. There are three procedures to obtain crisp T2FDPs in the singular data form. These procedures include fuzzification (alpha-cut operation), type-reduction and defuzzification processes. Upon carrying out these procedures, we use interpolating Bezier curve model to visualize the complex uncertainty data points denoted as type-2 fuzzy interpolation Bezier curve (T2FIBC).
\end{abstract}

Keywords: type-2 fuzzy set, type-2 fuzzy number, fuzzification, type-reduction, defuzzification, interpolation Bezier curve

\section{Introduction}

In CAD systems and computer graphics, data points in the form of control points are used to develop desired shapes using various types of curves and surfaces function. These curve and surface functions represent data points, which are used to analyze, manipulate and make a decision on developing a desired shape.

Problems may arise during data point collection from physical mock-up models, visualization from 3D scanner or Coordinate-measuring Machine (CMM), which leads to data uncertainty. This may occur due to measurement errors or device malfunction. Hence, the process of representing collected data points into curve and surface functions may lead to a different shape altogether. This may also result an undesired shape representation.

There are two options to overcom this problem. Either uncertainty data points are redefined manually to avoid uncertainty in data points or only use data points which are verified by comparing virtual models with physical mock-ups. Although we could design curves and surfaces by using verified data points, but this method is not just time consuming but the model does not capture the essence of the physical mock-up since the uncertainty data points were avoided.

Zadeh introduced type-1 fuzzy sets theory (T1FST) to solve uncertainty problem (Zadeh, 1965). Since the establishment of T1FST, there are many theories and concepts were introduced to solve many problems involving uncertain data. One of the concepts is called type-1 fuzzy number (T1FN) concepts (Dubois \& Prade, 1980; Klir \& Yuan, 1995; Zimmermann, 1985), which deals with the uncertainty of real values. This concept is used in CAGD to define the uncertainty data points as type-1 fuzzy data points (T1FDPs) which is elaborated further below.

In order to design various shapes with T1FDPs, T1FDPs are substituded with control points in the curve and surface functions. These functions can be found in (Farin, 1999, 2002; Farin, Hoschek, \& Kim, 2002; Piegl \& Tiller, 1995; Rogers, 2001; Salomon, 2006; Yamaguchi, 1988). When T1FDPs are being modeled through curves and surfaces, then these circumstances called type-1 fuzzy curve and surface. The type- 1 fuzzy curves and surfaces model has been developed recently due to the requirement in modeling the T1FDPs which gives the illustration of the T1FDPs properties (Abbasbandy, 2001; Abbasbandy \& Babolian, 1998; Abbasbandy, Ezzati, \& Behforooz, 2008; Abd. Fatah \& Rozaimi, 2012; Abd. Fatah, Jamaluddin, \& Ahmad, 2009; Abd. Fatah, Jamaluddin, Ahmad, \& Abu Osman, 2004; Abd. Fatah, Rozaimi, \& Jamaluddin, 2010; Anile, Falcidieno, Gallo, Spagnuolo, \& Spinello, 2000; Behforooz, Ezzati, \& Abbasbandy, 2010; Gallo \& Spagnuolo, 1998; Gallo, 
Spagnuolo, \& Spinello, 1998, 2000; Lodwick, 2008; Nurul Ain, Abd Fatah, Gobithaasan, \& Rozaimi, 2013; Rozaimi \& Abd. Fatah, 2010, 2012; Rozaimi, Abd. Fatah, \& Jamaluddin, 2010a, 2010b).

However, the T1FST method reaches its limit when the uncertainty data points become more complex. Therefore, we need a new higher-level definition of T1FST in defining the complex uncertainty data points. In 1975, Zadeh introduced the type-2 fuzzy set theory (T2FST) which used to solve complex uncertainty matter, including in defining the complex uncertainty data points.

This paper discusses about the construction of T2FDPs from complex uncertainty data points based on type-2 fuzzy number (T2FN) concept. In this paper, uncertainty data points are defined as type-2 fuzzy data points (T2FDPs). Three T2FDPs procedures are applied to obtain crisp T2FDPs solution as the final result. These procedures are the fuzzification, type-reduction and defuzzification processes, which defined based on the formulation of T2FDPs. To illustrate its application, we develop interpolating Bezier curve model by means of T2FDPs, which is denoted as T2FIBC.

This paper begins with Section 2, which discusses about the fundamental of T2FST, T2FN, and T2FR. These concepts are used to define the T2FDPs. For Section 3 defines T2FDPs from complex uncertainty data points together with the definitions of fuzzification (alpha-cut operation), type-reduction and defuzzification processes against T2FDPs. Section 4 elaborates on how interpolating cubic Bezier curve model is constructed. It is then followed by the construction of interpolating Bezier curve mnodel with T2FDPs denoted as T2FICBC model. Section 5 further elaborates the fuzzification, type-reductionand defuzzification processes involved to obtain crisp T2FICBC solution. Section 6-concludes the findings of this research.

\section{Preliminaries}

This section discusses about the fundamentals of T2FST, T2FN and also type- 2 fuzzy relation (T2FR). These definitions are used in defining the complex uncertainty data points in the next section.

Definition 1. A type-2 fuzzy set (T2FS), denoted $\overrightarrow{\vec{A}}$, is characterized by a type-2 membership function $\mu_{\ddot{\ddot{A}}}(x, u)$, where $x \in X$ and $u \in U_{x} \subseteq[0,1]$ that is,

$$
\vec{A}=\left\{\left((x, u), \mu_{\ddot{\ddot{A}}}(x, u)\right) \mid \forall x \in X, \forall u \in U_{x} \subseteq[0,1]\right\}
$$

in which, $0 \leq \mu_{\ddot{\ddot{A}}}(x, u) \leq 1 \quad$ (Mendel, 2001).

Definition 2. A T2FN is broadly defined as a T2FS that has a numerical domain. An interval T2FS is defined using the following four constraints, where $\overrightarrow{\vec{A}}_{\alpha}=\left\{\left[a^{\alpha}, b^{\alpha}\right],\left[c^{\alpha}, d^{\alpha}\right]\right\}, \forall \alpha \in[0,1], \forall a^{\alpha}, b^{\alpha}, c^{\alpha}, d^{\alpha} \in \mathbb{R}$ (Figure 1) (Aguero \& Vargas, 2007):

1) $a^{\alpha} \leq b^{\alpha} \leq c^{\alpha} \leq d^{\alpha}$

2) $\left[a^{\alpha}, d^{\alpha}\right]$ and $\left[b^{\alpha}, c^{\alpha}\right]$ generate a function that is convex and $\left[a^{\alpha}, d^{\alpha}\right]$ generate a function is normal.

3) $\forall \alpha_{1}, \alpha_{2} \in[0,1]:\left(\alpha_{2}>\alpha_{1}\right) \Rightarrow\left(\left[a^{\alpha_{1}}, c^{\alpha_{1}}\right] \supset\left[a^{\alpha_{2}}, c^{\alpha_{2}}\right],\left[b^{\alpha_{1}}, d^{\alpha_{1}}\right] \supset\left[b^{\alpha_{2}}, d^{\alpha_{2}}\right]\right)$, for $c^{\alpha_{2}} \geq b^{\alpha_{2}}$.

4) If the maximum of the membership function generated by $\left[b^{\alpha}, c^{\alpha}\right]$ is the level $\alpha_{m}$, that is, $\left[b^{\alpha_{m}}, c^{\alpha_{m}}\right]$, then $\left[b^{\alpha_{m}}, c^{\alpha_{m}}\right] \subset\left[a^{\alpha=1}, d^{\alpha=1}\right]$.
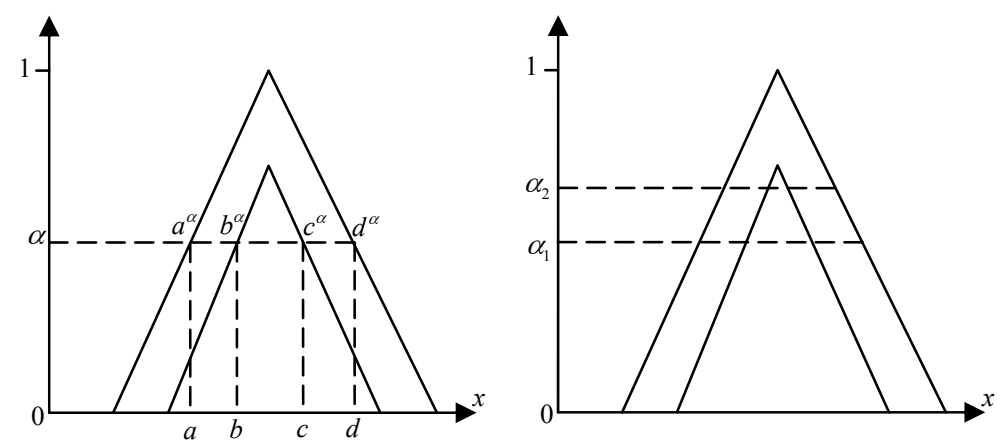

Figure 1. The illustration for definition of an interval T2FN 
Definition 3. A type-2 fuzzy relation (T2FR) is a T2FS defined on the Cartesian product of crisp sets $X_{1}, X_{2}, \ldots, X_{n}$ where the tuples $\left(x_{1}, x_{2}, \ldots, x_{n}\right)$ have varying degree of membership which are type-1 fuzzy sets (T1FSs) (John \& Lake, 2001).

\section{Type-2 Fuzzy Data Points}

This Section 3 discusses about the construction of T2FDPs based on the definitions which were given in Section 2. This definition of brand new T2FR is based on the T1FR refers to (Zimmermann, 1985) for detail.

Definition 4. Let $X, Y, U_{x}, V_{y} \subseteq R$ and

$$
\begin{gathered}
\overrightarrow{\vec{A}}=\left\{\left((x, u), \mu_{\ddot{\ddot{A}}}(x, u)\right) \mid \forall x \in X, \forall u \in U_{x} \subseteq[0,1]\right\} \text { and } \\
\overrightarrow{\vec{B}}=\left\{\left((y, v), \mu_{\ddot{\ddot{B}}}(y, v)\right) \mid \forall y \in Y, \forall v \in V_{y} \subseteq[0,1]\right\}
\end{gathered}
$$

are two T2FSs. Then, $\quad \stackrel{\vec{R}}{R}=\left\{\left(((x, u),(y, v)), \quad \mu_{\ddot{\ddot{R}}}\left(\mu_{\ddot{\ddot{A}}}(x, u), \mu_{\ddot{\ddot{B}}}(y, v)\right)\right) \mid \quad\left(\forall x \in X, \forall u \in U_{x}\right)\right.$ $\left.\times\left(\forall y \in Y, \forall u \in V_{y}\right) \subseteq[0,1]\right\} \quad$ is $\quad$ a $\quad \mathrm{T} 2 \mathrm{FR} \quad$ on $\quad \overrightarrow{\vec{A}} \quad$ and $\quad \overrightarrow{\vec{B}} \quad$ if $\quad \mu_{\ddot{\vec{R}}}\left(\mu_{\ddot{\ddot{A}}}(x, u), \mu_{\ddot{\ddot{B}}}(y, v)\right) \leq \mu_{\ddot{A}}(x, u)$, $\forall((x, u),(y, v)) \in\left(\forall x \in X, \forall u \in U_{x}\right) \times\left(\forall y \in Y, \forall v \in V_{y}\right)$.

Definition 5. Let $P=\{x \mid x$ type-2 fuzzy point $\}$ and $\stackrel{\vec{P}}{=}=\left\{P_{i} \mid P_{i}\right.$ data point $\}$ which is set of type-2 fuzzy data point with $P_{i} \in P \subset X$, where $X$ is a universal set and $\mu_{P}\left(P_{i}\right): P \rightarrow[0,1]$ is the membership function which defined as $\mu_{P}\left(P_{i}\right)=1$ and formulated by $\vec{P}=\left\{\left(P_{i}, \mu_{P}\left(P_{i}\right)\right) \mid P_{i} \in \mathbb{R}\right\}$. Therefore,

$$
\mu_{P}\left(P_{i}\right)= \begin{cases}0 & \text { if } P_{i} \notin X \\ c \in(0,1) & \text { if } P_{i} \in X \\ 1 & \text { if } P_{i} \in X\end{cases}
$$

with $\mu_{P}\left(P_{i}\right)=\left\langle\mu_{P}\left(\vec{P}_{i}^{\leftarrow}\right), \mu_{P}\left(P_{i}\right), \mu_{P}\left(\vec{P}_{i}^{\rightarrow}\right)\right\rangle_{\leftarrow}$ which $\mu_{P}\left(\vec{P}_{i}^{\leftarrow}\right)$ and $\mu_{P}\left(\vec{P}_{i}^{\rightarrow}\right)$ are left and right footprint of membership values with $\mu_{P}\left(\vec{P}_{i}^{\leftarrow}\right)=\left\langle\mu_{P}\left(\vec{P}_{i}^{\leftarrow}\right), \mu_{P}\left(\vec{P}_{i}^{\leftarrow}\right), \mu_{P}\left(\vec{P}_{i}^{\leftarrow}\right)\right\rangle$ where, $\mu_{P}\left(\vec{P}_{i}^{\leftarrow}\right), \mu_{P}\left(\vec{P}_{i}^{\leftarrow}\right)$ and $\mu_{P}\left(\vec{P}_{i}^{\leftarrow}\right)$ are left-left, left, right-left membership grade values and $\mu_{P}\left(\vec{P}_{i}^{\leftarrow}\right)=\left\langle\mu_{P}\left(\vec{P}_{i}^{\leftarrow}\right), \mu_{P}\left(\vec{P}_{i}^{\leftarrow}\right), \mu_{P}\left(\vec{P}_{i}^{\leftarrow}\right)\right\rangle$ where $\mu_{P}\left(\vec{P}_{i}^{\rightarrow}\right)$, $\mu_{P}\left(\vec{P}_{i}^{\rightarrow}\right)$ and $\mu_{P}\left(\vec{P}_{i}^{\leftarrow}\right)$ are right-right, right, left-right membership grade values, which can be written as

$$
\overrightarrow{\vec{P}}=\left\{\vec{P}_{i}: i=0,1,2, \ldots, n\right\}
$$

for every $i, \overrightarrow{\vec{P}}_{i}=\left\langle\overrightarrow{\vec{P}}_{i}^{\leftarrow}, P_{i}, \overrightarrow{\vec{P}}_{i}^{\rightarrow}\right\rangle$, with $\overrightarrow{\vec{P}}_{i}^{\leftarrow} \underset{\rightarrow}{=}\left\langle\vec{P}_{i}^{\leftarrow}, \vec{P}_{i}^{\leftarrow}, \vec{P}_{i \leftarrow}^{\leftarrow}\right\rangle$ where $\vec{P}_{i}^{\leftarrow}, \vec{P}_{i}^{\leftarrow}$ and $\vec{P}_{i}^{\leftarrow} \quad$ are left-left, left and right-left T2FDPs and $\vec{P}_{i}^{\rightarrow}=\left\langle\vec{P}_{i}^{\rightarrow}, \vec{P}_{i}^{\rightarrow}, \vec{P}_{i}^{\rightarrow}\right\rangle$ where $\vec{P}_{i}^{\rightarrow}, \vec{P}_{i}^{\rightarrow}$ and $\stackrel{\vec{P}_{i}^{\rightarrow}}{\rightarrow}$ are left-right, right and right-right T2FDPs respectively. This can be illustrated as in Figure 2.

The illustration of T2FDP was shown in Figure 2 which T1FDP becomes the primary membership function bounded by upper bound, $\left[\vec{P}^{\leftarrow}, P, \vec{P}^{\rightarrow}\right]$ and lower bound, $\left[\vec{P}^{\leftarrow}, P, \vec{P}^{\rightarrow}\right]$ respectively. The process of defining T2FDP can be shown through Figure 3. 


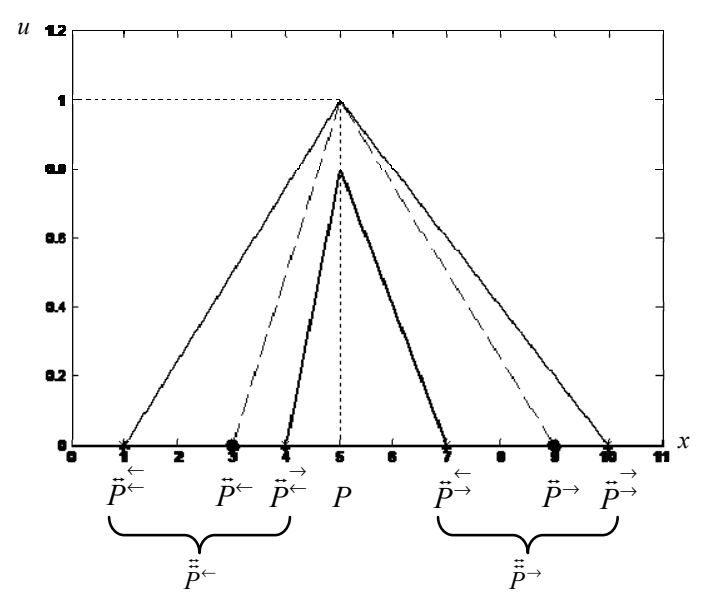

Figure 2. T2FDP around 5
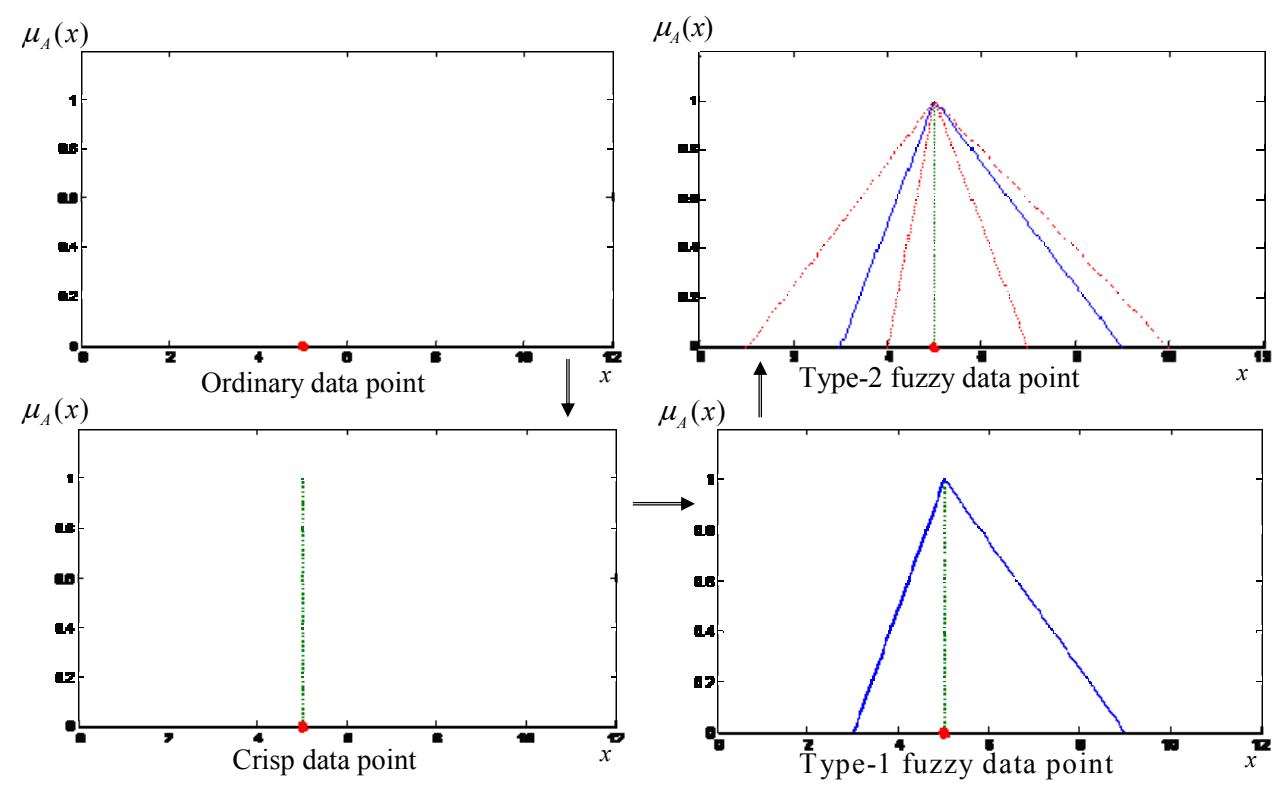

Figure 3. The difference between type-1 and type-2 fuzzy data point

Figure 3 shows that the process of defining T2FDP from the ordinary point. This T2FDP formed based on the definition of T2FN and T2FR.

The alpha-cut definition indicates how to obtain interval type-2 fuzzy data points (IT2FDPs) based on the selection of the alpha values (membership functions value). The operation of alpha-cut against the T2FDPs can be defined as follows.

Definition 6. Based on Def. 5, let $\overrightarrow{\ddot{P}}$ be the set of T2FDPs with $\overrightarrow{\ddot{P}}_{i} \in \vec{P}$ where $i=0,1, \ldots, n-1$. Then $\vec{P}_{\alpha}$ is the alpha-cut operation of T2FDPs which is given as equation as follows.

$$
\begin{aligned}
\overrightarrow{\vec{P}}_{i_{\alpha}} & =\left\langle\overrightarrow{\vec{P}}_{i_{\alpha}}^{\leftarrow}, P_{i}, \overrightarrow{\vec{P}}_{i_{\alpha}}^{\overrightarrow{ }}\right\rangle \\
& =\left\langle\left\langle\vec{P}_{i_{\alpha}}^{\leftarrow} ; \vec{P}_{i_{\alpha}}^{\leftarrow} ; \vec{P}_{i_{\alpha}}^{\leftarrow}\right\rangle, P_{i},\left\langle\vec{P}_{i_{\alpha}}^{\leftarrow} ; \vec{P}_{i_{\alpha}}^{\rightarrow} ; \vec{P}_{i_{\alpha}}^{\rightarrow}\right)\right\rangle \\
& =\left\langle\left[\left(P_{i}-\left\langle\vec{P}_{i}^{\leftarrow} ; \vec{P}_{i}^{\leftarrow} ; \vec{P}_{i}^{\leftarrow}\right\rangle\right) \alpha+\left\langle\vec{P}_{i}^{\leftarrow} ; \vec{P}_{i}^{\leftarrow} ; \vec{P}_{i}^{\leftarrow}\right\rangle\right], P_{i},\left[-\left(\left\langle\vec{P}_{i}^{\leftarrow} ; \vec{P}_{i}^{\rightarrow} ; \vec{P}_{i}^{\rightarrow}\right\rangle-P_{i}\right) \alpha+\left\langle\vec{P}_{i}^{\leftarrow} ; \vec{P}_{i}^{\rightarrow} ; \vec{P}_{i}^{\rightarrow}\right\rangle\right]\right\rangle
\end{aligned}
$$

This alpha-cut definition illustrated in Figure 4. 


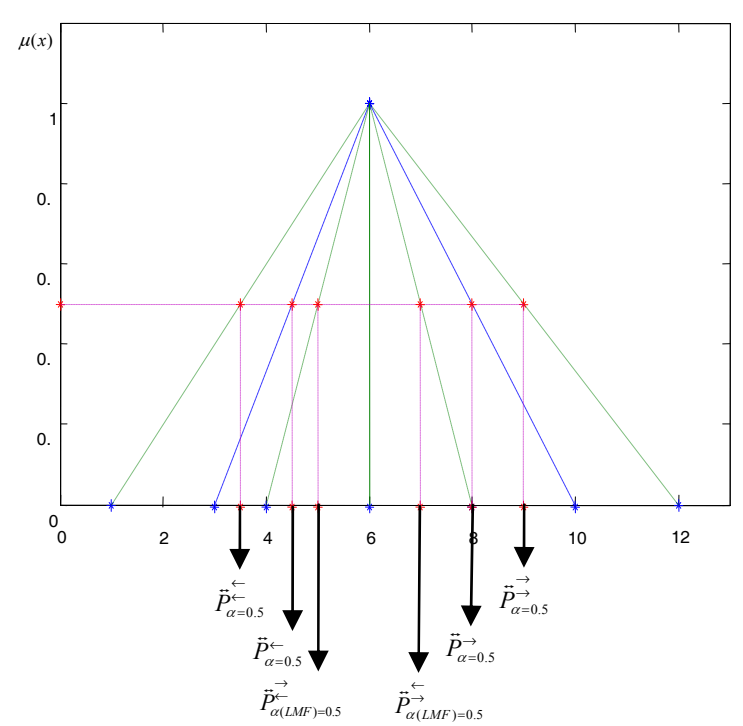

Figure 4. The alpha-cut operation towards T2FDPs

The alpha-cut process which implies to T2FDPs is known as fuzzification process of T2FDPs. This process is the first process in order to obtain the crisp T2FDPs solution which gives the IT2FDPs based on the alpha values that was prescribed, $\alpha \in(0,1]$. The next process is type-reduction process sated above IT2FDPs are being reduced to IT1FDPs for allowing us to carry out defuzzification process of type-1 fuzzy case. This process is the final step in getting the crisp T2FDPs solution. Type-reduction dan defuzzification processes defined as follows.

Definition 7. Let $\overrightarrow{\vec{P}}_{i}$ be a set $(n+1)$ T2FDPs, then the type-reduction method of $\alpha$-T2FDPs (after fuzzification), $\overline{\vec{P}}_{i_{\alpha}}$ is defined by

$$
\overline{\vec{P}}_{\alpha}=\left\{\overline{\vec{P}}_{i_{\alpha}}=\left\langle\overline{\vec{P}}_{i_{\alpha}}^{\leftarrow}, P_{i}, \overline{\vec{P}}_{i_{\alpha}}^{\rightarrow}\right\rangle ; i=0,1, \ldots, n\right\}
$$

where $\overline{\vec{P}}_{i_{\alpha}}^{\leftarrow}$ is left type-reduction of $\alpha$-cut T2FDPs, $\overline{\vec{P}}_{i_{\alpha}}^{\leftarrow}=\frac{1}{3} \sum_{i=0, \ldots, n}\left\langle\vec{P}_{i_{\alpha}}^{\leftarrow}+\vec{P}_{i_{\alpha}}^{\leftarrow}+\vec{P}_{i_{\alpha}}^{\leftarrow}\right\rangle, P_{i}$ is the crisp point and $\overrightarrow{\vec{P}}_{i_{\alpha}}^{\rightarrow}$ is right type-reduction of $\alpha$-cut T2FDPs, $\quad \bar{P}_{i_{\alpha}}^{\rightarrow}=\frac{1}{3} \sum_{i=0, \ldots, n}\left\langle\stackrel{\leftarrow}{\vec{P}_{i_{\alpha}}}+\vec{P}_{i_{\alpha}}^{\rightarrow}+\vec{P}_{i_{\alpha}}^{\rightarrow}\right\rangle$.

Definition 8. Let $\alpha$-TR is the type-reduction method after alpha-cut process had been applied for every existing T2FDPs, $\overline{\vec{P}}_{i_{\alpha}}$. Then, $\overline{\bar{P}}_{i_{\alpha}}$ is denoted as defuzzification of T2FDPs for $\overline{\vec{P}}_{i_{\alpha}}$ if for every $\overline{\vec{P}}_{i_{\alpha}} \in \overrightarrow{\vec{P}}$,

$$
\overline{\bar{P}}_{\alpha}=\left\{\overline{\bar{P}}_{i_{\alpha}}\right\} \quad \text { for } \quad i=0,1, \ldots, n
$$

where for every $\overline{\bar{P}}_{i_{\alpha}}=\frac{1}{3} \sum_{i=0}<\overline{\vec{P}}_{i_{\alpha}}^{\leftarrow}, P_{i}, \overline{\vec{P}}_{i_{\alpha}}^{\rightarrow}>$. The process in defuzzifying of T2FDPs is depicted in Figure 5 . 


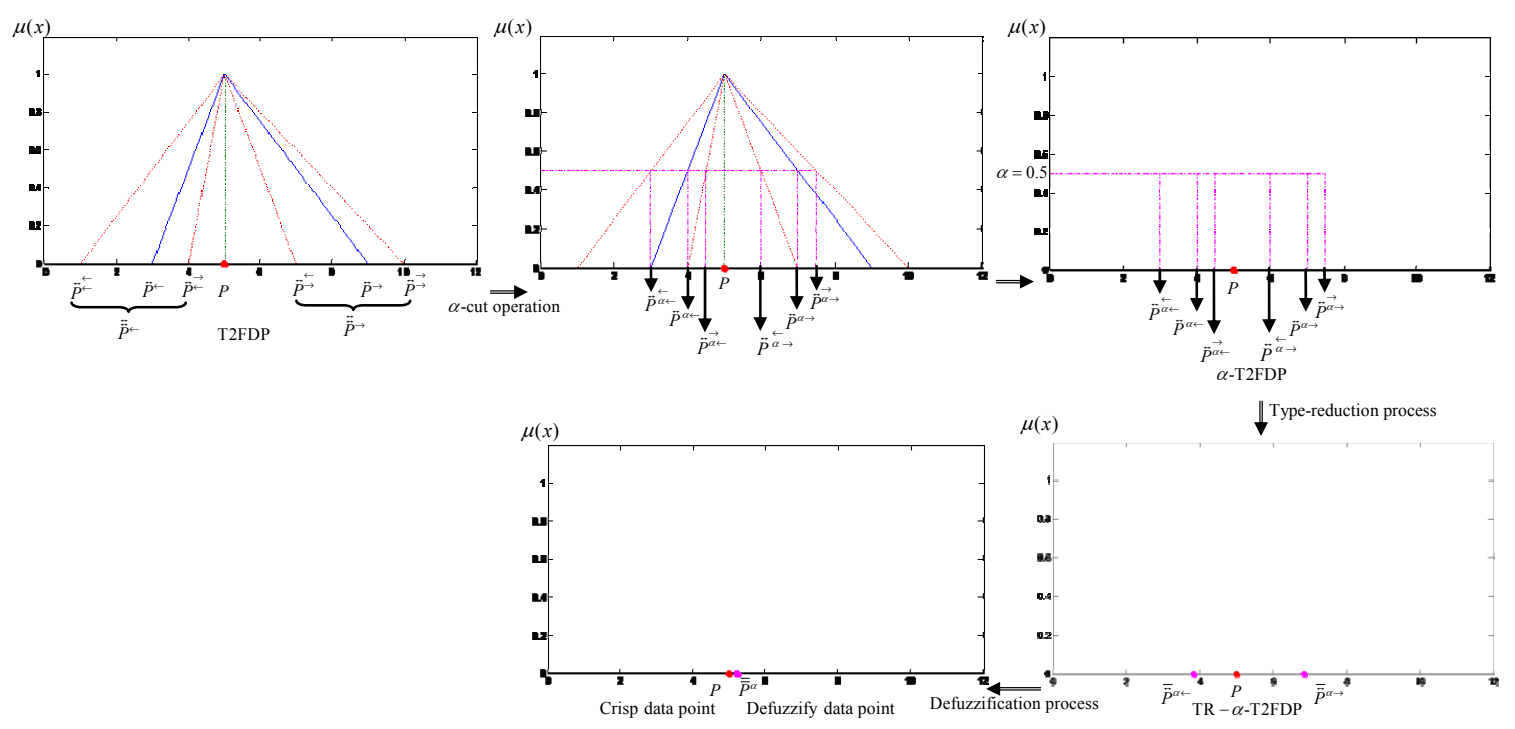

Figure 5. Defuzzification process of T2FDP

\section{Interpolation Bezier Curve Model}

This section will discuss about the construction of interpolating Bezier curve model. In this case, we choose the cubic form for the interpolation curve model for piecewise formation which permits $c$ and $s$ design (Gobithasan $\&$ Ali, 2004). Therefore, the interpolating Bezier curve model follow suit;

Definition 9. Let $P_{i}, D_{i} \in B, i=0,1, \ldots, n$ be a given set of data points and the derivative value at $t$. Then, the interpolation cubic Bezier curve is defined (Sarfraz \& Razzak, 2002) as

which $K_{i}$ and $L_{i}$ are

$$
B(t)=(1-t)^{3} P_{i}+3 t(1-t)^{2} K_{i}+3 t^{2}(1-t) L_{i}+t^{3} P_{i+1}
$$

$$
\begin{aligned}
& K_{i}=\frac{D_{i}}{3}+P_{i} \\
& L_{i}=P_{i+1}-\frac{D_{i+1}}{3}
\end{aligned}
$$

with $D_{i}$ and $D_{i+1}$ are tangent vectors at $P_{i}$ and $P_{i+1}$ respectively. These tangent vectors are defined as follows. For open curves,

$$
\begin{aligned}
& D_{0}=2\left(P_{1}-P_{0}\right)-\frac{P_{2}-P_{0}}{2}, \\
& D_{i}=a_{i}\left(P_{i}-P_{i+1}\right)+\left(1-a_{i}\right)\left(P_{i+1}-P_{i}\right), \\
& D_{n}=2\left(P_{n}-P_{n-1}\right)-\frac{P_{n}-P_{n-2}}{2},
\end{aligned}
$$

where

$$
a_{i}=\frac{\left\|P_{i+1}-P_{i}\right\|}{\left\|P_{i+1}-P_{i}\right\|\left\|P_{i}-P_{i-1}\right\|}, \quad i=0,1, \ldots, n .
$$

Thus, the curves lies in the convex hull of control points $\left\{P_{i}, K_{i}, L_{i}, P_{i+1}\right\}$ and variation diminishing property is also satisfied (Bernstein Bezier Theory). 


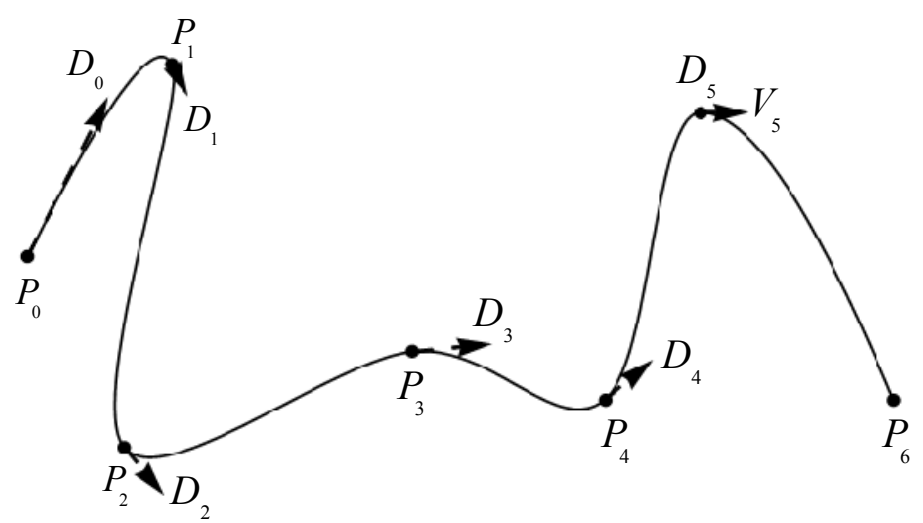

Figure 6. Interpolating cubic Bezier curve with $D_{0}, D_{1}, \ldots, D_{5}$ as the tangent vectors

Figure 6 illustrates data points, $P_{0}, P_{1}, \ldots, P_{6}$ and their tangent vectors, $D_{0}, D_{1}, \ldots, D_{5}$ respectively were interpolated with interpolating cubic Bezier curve with smoothness as $C^{1}$-continuity.

\section{Type-2 Fuzzy Data Points with Interpolating Bezier Curve Model}

In this chapter, we blend T2FDPs and interpolating Bezier curve together to form type-2 fuzzy interpolation Bezier curve model.

Definition 10. Let $\overrightarrow{\vec{P}}_{i}, \quad \overrightarrow{\vec{D}} \in \overrightarrow{\vec{B}}, i=0,1, \ldots, n$ be a given set of T2FDPs and the derivative value at $t$. Then, the T2FICBC is defined as

$$
\overrightarrow{\vec{B}}(t)=(1-t)^{3} \vec{P}_{i}+3 t(1-t)^{2} \overrightarrow{\vec{K}}_{i}+3 t^{2}(1-t) \overrightarrow{\vec{L}}_{i}+t^{3} \vec{P}_{i+1}
$$

where $\overrightarrow{\vec{K}}_{i}$ and $\overrightarrow{\vec{L}}_{i}$ defined as

$$
\begin{aligned}
& \overrightarrow{\tilde{K}}_{i}=\frac{\overrightarrow{\vec{D}}_{i}}{3}+\vec{P}_{i} \\
& \overrightarrow{\vec{L}}_{i}=\overrightarrow{\vec{P}}_{i+1}-\frac{\overrightarrow{\vec{D}}_{i+1}}{3}
\end{aligned}
$$

with $\overrightarrow{\ddot{D}}_{i}$ and $\overrightarrow{\tilde{D}}_{i+1}$ are type-2 fuzzy tangent vectors at $\overrightarrow{\ddot{P}}_{i}$ and $\overrightarrow{\ddot{P}}_{i+1}$ respectively. These type-2 fuzzy tangent vectors are defined as follow. For type-2 fuzzy open curves,

$$
\begin{aligned}
& \overrightarrow{\vec{D}}_{0}=2\left(\overrightarrow{\vec{P}}_{1}-\overrightarrow{\vec{P}}_{0}\right)-\frac{\vec{P}_{2}-\vec{P}_{0}}{2}, \\
& \overrightarrow{\vec{D}}_{i}=a_{i}\left(\overrightarrow{\vec{P}}_{i}-\overrightarrow{\vec{P}}_{i+1}\right)+\left(1-a_{i}\right)\left(\overrightarrow{\vec{P}}_{i+1}-\overrightarrow{\vec{P}}_{i}\right), \\
& \overrightarrow{\vec{D}}_{n}=2\left(\overrightarrow{\vec{P}}_{n}-\overrightarrow{\vec{P}}_{n-1}\right)-\frac{\vec{P}_{n}-\overrightarrow{\vec{P}}_{n-2}}{2},
\end{aligned}
$$

where

$$
a_{i}=\frac{\left\|\vec{P}_{i+1}-\vec{P}_{i}\right\|}{\left\|\vec{P}_{i+1}-\vec{P}_{i}\right\|\left\|\overrightarrow{\vec{P}}_{i}-\vec{P}_{i-1}\right\|}, \quad i=0,1, \ldots, n .
$$

Thus, the type-2 fuzzy curves lies in the type-2 fuzzy convex hull of type-2 fuzzy control points $\left\{\ddot{P}_{i}, \vec{K}_{i}, \vec{L}_{i}, \ddot{P}_{i+1}\right\}$ and satisfying its variation diminishing (Bernstein Bezier theory). 
The T2FICBC model has been defined based on Def. 10. Figure 7 depicts interpolating cubic Bezier curve model defined in Def. 10.

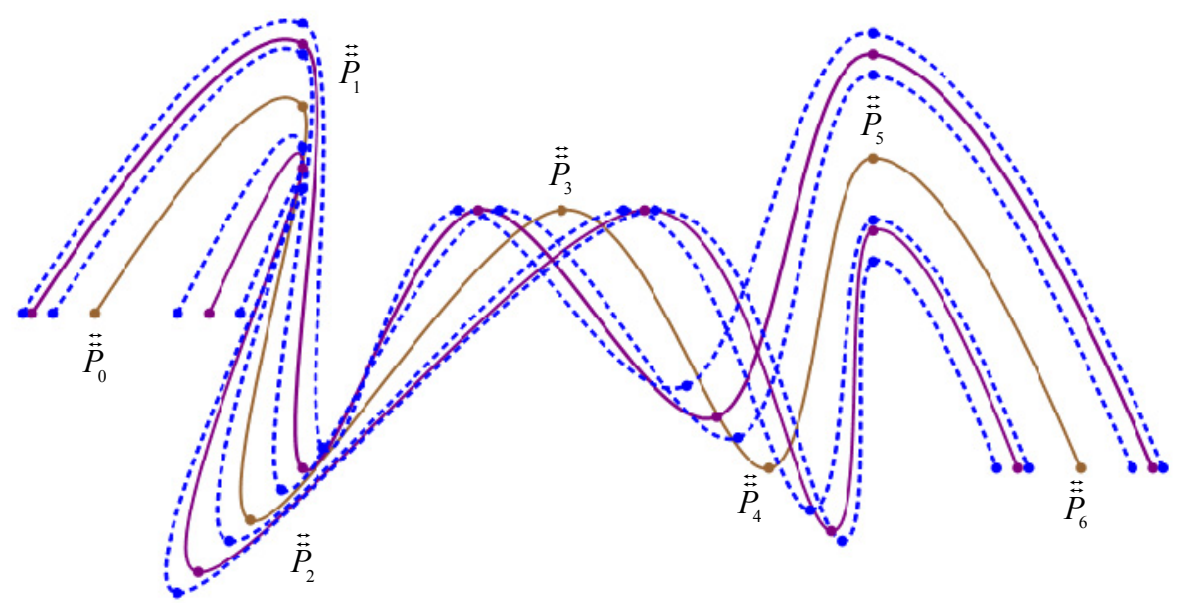

Figure 7. The T2FDPs has been modeled by interpolation cubic Bezier curve model

Up to now, T2FDPs has been defined from complex uncertainty data by means of T2FST, T2FN, T2FR and interpolating cubic Bezier curve model. T2FDPs are represented in a curve form as T2FICBC. This type-2 curve called T2FICBC due to the blending of every T2FDPs which formed by the type-2 fuzzy interval, $\overrightarrow{\vec{P}}_{i}=\left[\vec{P}_{i}^{\leftarrow}, \vec{P}_{i}^{\leftarrow}, \vec{P}_{i}^{\leftarrow}, P_{i}, \vec{P}_{i}^{\rightarrow}, \vec{P}_{i}^{\rightarrow}, \vec{P}_{i}^{\leftarrow}\right]$.

Next step involves three processes namely fuzzification, type-reduction and defuzzification processes which are explained below:

Algorithm 1. Algorithm of fuzzification, type-reduction and defuzzification processes of T2FICBC.

Step 1: Define T2FDPs.

For example, six T2FDP, $\vec{P}_{i=0, \ldots, 6}$. Then, based on Def. 5 and Def. 10, T2FICBC may result curves as depicted in Figure 7.

Step 2: Fuzzification process (alpha-cut operation).

For left( $x$-axis $) /$ lower(y-axis),

$$
\left(\ddot{\vec{P}}_{i=0, \ldots, 6}^{\leftarrow}-P_{i=0, \ldots, 6}\right) \alpha_{0.5}+\ddot{P}_{i=0, \ldots, 6}^{\leftarrow} \text { where } \ddot{P}_{i=0, \ldots, 6}^{\leftarrow}=\left\langle\vec{P}_{i}^{\leftarrow}, \vec{P}_{i}^{\leftarrow}, \vec{P}_{i}^{\leftarrow}\right\rangle_{i=0, \ldots, 6}
$$

For right(x-axis)/upper(y-axis),

$$
\left(\ddot{\vec{P}}_{i=0, \ldots, 6}^{\rightarrow}-P_{i=0, \ldots, 6}\right) \alpha_{0.5}+\vec{P}_{i=0, \ldots, 6}^{\rightarrow} \text { where } \vec{P}_{i=0, \ldots, 6}^{\rightarrow}=\left\langle\vec{P}_{i}^{\stackrel{\leftarrow}{\rightarrow}}, \vec{P}_{i}^{\rightarrow}, \vec{P}_{i}^{\rightarrow}\right\rangle_{i=0, \ldots, 6} .
$$

Then, the new T2FDPs $(\alpha$-T2FDPs) were modeled through the interpolation cubic Bezier curve model which gives $\alpha$-T2FICBC based on Def. 10 and illustrated by Figure 8 .

$$
\overrightarrow{\vec{B}}_{\alpha}(t)=(1-t)^{3} \vec{P}_{i_{\alpha}}+3 t(1-t)^{2} \overrightarrow{\tilde{K}}_{i_{\alpha}}+3 t^{2}(1-t) \overrightarrow{\vec{L}}_{i_{\alpha}}+t^{3} \vec{P}_{i+1_{\alpha}},
$$




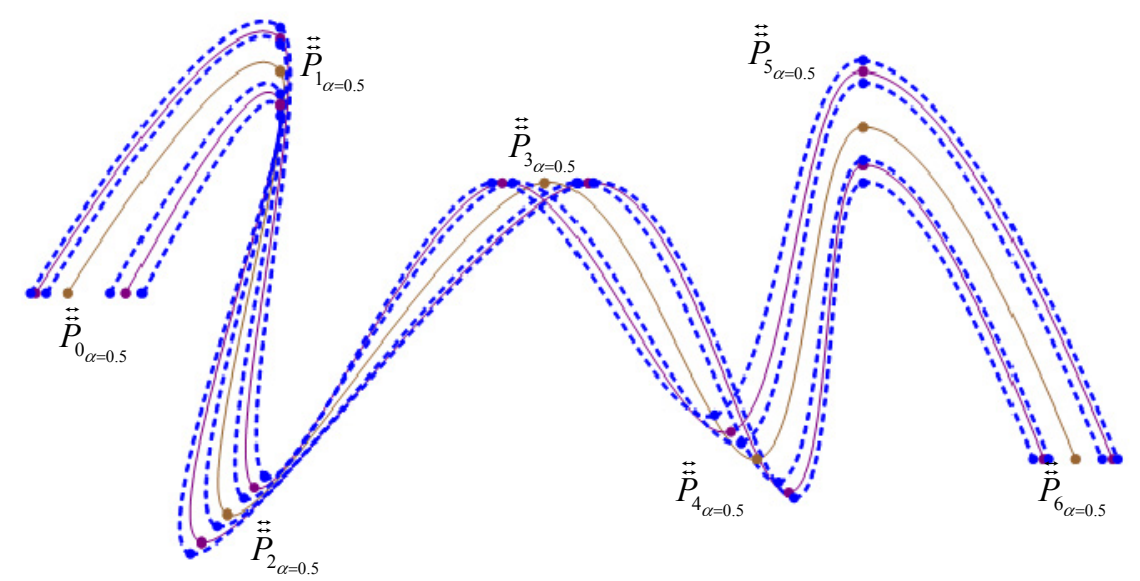

Figure 8 . The new T2FICBC( $\alpha$-T2FICBC) after fuzzification $\operatorname{process}(\alpha$-cut operation)

Step 3: Type-reduction process of $\alpha$-T2FICBC.

Based on Def. 7, the type-reduction equation of $\alpha$-T2FICBC can be given as follows

$$
\begin{aligned}
\overline{\vec{P}}_{i=0, \ldots, 6_{\alpha}} & =\left\langle\sum_{i=0, \ldots, 6} \frac{\left\langle\vec{P}_{i_{\alpha}}^{\leftarrow}+\vec{P}_{i_{\alpha}}^{\leftarrow}+\vec{P}_{i_{\alpha}}^{\leftarrow}\right\rangle}{3}, P_{i}, \sum_{i=0, \ldots, 6} \frac{\left\langle\vec{P}_{i_{\alpha}}^{\leftarrow}+\vec{P}_{i_{\alpha}}^{\rightarrow}+\vec{P}_{i_{\alpha}}^{\rightarrow}\right.}{3}\right\rangle \\
& =\left\langle\overline{\vec{P}}_{i_{\alpha}}^{\leftarrow}, P_{i}, \overrightarrow{\vec{P}}_{i_{\alpha}}^{\rightarrow}\right\rangle .
\end{aligned}
$$

The type-reduction of $\alpha$-T2FICBC(TR- $\alpha$-T2FICBC) can be written as follows.

$$
\overline{\vec{B}}_{\alpha}(t)=(1-t)^{3} \overline{\vec{P}}_{i_{\alpha}}+3 t(1-t)^{2} \overline{\vec{K}}_{i_{\alpha}}+3 t^{2}(1-t) \overline{\vec{L}}_{i_{\alpha}}+t^{3} \overline{\vec{P}}_{i+1_{\alpha}},
$$

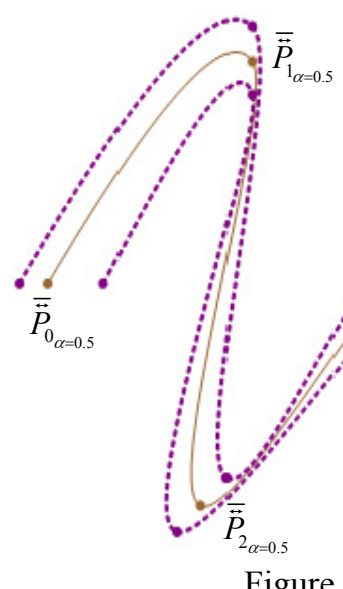

Figure 9. The type-reduce $\alpha$-T2FICBC modeling

Step 4: The defuzzification process

The defuzzification process of TR- $\alpha$-T2FICBC involve the following formula.

$$
\begin{aligned}
\overline{\bar{P}}_{i=0, \ldots, 6_{\alpha}}= & \sum_{i=0, \ldots, 6} \frac{\left\langle\overline{\vec{P}}_{i_{\alpha}}^{\leftarrow}+P_{i}+\overline{\vec{P}}_{i_{\alpha}}^{\rightarrow}\right\rangle}{3} \\
= & \overline{\bar{P}}_{i=0, \ldots, 3} .
\end{aligned}
$$

Then, the crisp T2FICBC solution model is given by Figure 10 . 


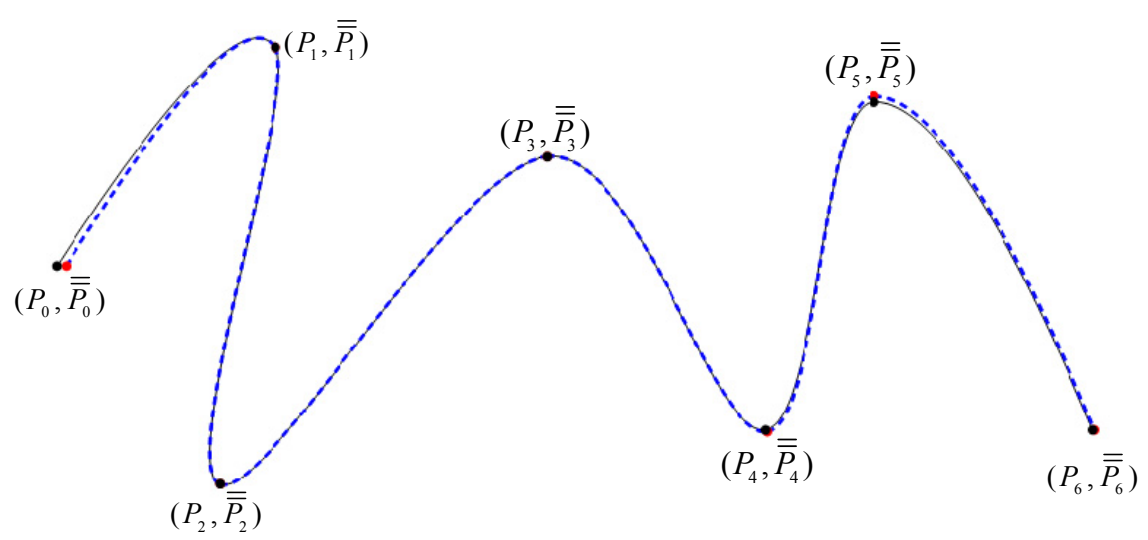

Figure 10. Defuzzified curve obtained with TR- $\alpha$-T2FICBC

Figure 10 shows the crisp T2FICBC solution obtained after defuzzification process. The crisp T2FICBC is being modeled with the crisp interpolation Bezier curve to simply shows the difference between those data points and also can make a conclusion based on the modeling T2FDPs and crisps data points.

\section{Conclusion}

The steps involved to develop type-2 fuzzy interpolation cubic Bezier curve (T2FICBC) has been shown along with simple numerical example. T2FDPs has been constructed by blending interpolating cubic Bezier curve model with the T2FDPs.

The proposed curve modeling method take into consideration all the uncertainty data to produce a probable curve based on alpha-cut. There are also other processes implemented to obtain crisp T2FDPs solution where T2FDPs become a singular data point (upon data points defuzzification). The processes are fuzzification (alpha-cut operation) to obtain the T2FDPs interval based on the alpha values, type-reduction process which reduced T2FDPs to becomes T1FDPs for allowing the defuzzfication process of type- 1 . The process is the defuzzification process which is used to obtain the crisp T2FDPs solution in a singular form which this process became the final process for obtaining crisp T2FDPs solution.

\section{Acknowledgements}

The authors would like to thank Research Management and Innovation Centre (RMIC) of Universiti Malaysia Terengganu and Ministry of Higher Education (MOHE) Malaysia for funding (FRGS, vot59244) and providing the facilities to carry out this research.

\section{References}

Abbasbandy, S. (2001). Interpolation of fuzzy data by complete splines. Journal of Applied Mathematics and Computing, 8(3), 587-594.

Abbasbandy, S., \& Babolian, E. (1998). Interpolation of fuzzy data by natural splines. Journal of Applied Mathematics and Computing, 5(2), 457-463.

Abbasbandy, S., Ezzati, R., \& Behforooz, H. (2008). Interpolation of Fuzzy Data by Using Fuzzy splines. International Journal of Uncertainty, Fuzziness and Knowledge-Based Systems, 16(1), 107-115. http://dx.doi.org/10.1142/S0218488508005078

Abd. Fatah, W., \& Rozaimi, Z. (2012). Fuzzy Interpolation Rational Cubic Bezier Curves Modeling of Blurring Offline Handwriting Signature with Different Degree of Blurring. Applied Mathematical Sciences, 6(81), 4005-4016.

Abd. Fatah, W., Jamaluddin, M. A., \& Ahmad, A. M. (2009). Fuzzy Geometric Modeling. Paper presented at the Proceedings. International Conference on Computer Graphics, Imaging and Visualization, 2009. CGIV 2009.

Abd. Fatah, W., Jamaluddin, M. A., Ahmad, A. M., \& Abu Osman, M. T. (2004). Fuzzy Set In Geometric Modeling. Paper presented at the Proceedings. International Conference on Computer Graphics, Imaging and Visualization, 2004. CGIV 2004. http://dx.doi.org/ 10.1109/CGIV.2004.1323990

Abd. Fatah, W., Rozaimi, Z., \& Jamaluddin, M. A. (2010). Fuzzy Interpolation Rational Bezier Curve. Paper 
presented at the Seventh International Conference on Computer Graphics, Imaging and Visualization (CGIV 2010). http://dx.doi.org/10.1109/CGIV.2010.17

Aguero, J. R., \& Vargas, A. (2007). Calculating Functions of Interval Type-2 Fuzzy Numbers for Fault Current $\begin{array}{llllll}\text { Analysis. IEEE Transactions on } & \text { Fuzzy }\end{array}$ http://dx.doi.org/10.1109/TFUZZ.2006.889757

Anile, A. M., Falcidieno, B., Gallo, G., Spagnuolo, M., \& Spinello, S. (2000). Modeling uncertain data with $\begin{array}{lllll}\text { fuzzy B-splines. Fuzzy Sets and } & \text { Systems, }\end{array}$ http://dx.doi.org/10.1016/S0165-0114(98)00146-8

Behforooz, H., Ezzati, R., \& Abbasbandy, S. (2010). Interpolation of fuzzy data by using E(3) Cubic Splines. International Journal of Pure and Applied Mathematics, 60(4), 383-392.

Dubois, D., \& Prade, H. (1980). Fuzzy Sets and Systems: Theory and Applications. New York: Academic Press.

Farin, G. (1999). NURBS for Curve and Surface Design: from Projective Geometry to Practical Use (2nd ed.). AK Peters, Ltd.

Farin, G. (2002). Curves and Surfaces for CAGD: A Practical Guide (5th ed.). USA: Academic Press.

Farin, G., Hoschek, J., \& Kim, M. S. (2002). Handbook of Computer Aided Geometric Design. The Netherlands: Elsevier Science B.V.

Gallo, G., \& Spagnuolo, M. (1998). Uncertainty coding and controlled data reduction using fuzzy-B-splines. Paper presented at the Conference on Computer Graphics International, 1998. http://dx.doi.org/10.1109/CGI.1998.694307

Gallo, G., Spagnuolo, M., \& Spinello, S. (1998). Rainfall Estimation from Sparse Data with Fuzzy B-Splines. Journal of Geographic Information and Decision Analysis, 2(2), 194-203.

Gallo, G., Spagnuolo, M., \& Spinello, S. (2000). Fuzzy B-Splines: A Surface Model Encapsulating Uncertainty. Graphical Models, 62(1), 40-55. http://dx.doi.org/10.1006/gmod.1999.0512

Gobithasan, R., \& Ali, J. M. (2004). Towards G2 curve design with Timmer Parametric Cubic. Proceedings International Conference on Computer Graphics, Imaging and Visuualization, CGIV 2004, 109-114.

John, R., \& Lake, S. (2001). Type-2 fuzzy sets for modelling nursing intuition. Paper presented at the IFSA World Congress and 20th NAFIPS International Conference, 2001. Joint 9th. http://dx.doi.org/10.1109/NAFIPS.2001.944360

Klir, G. J., \& Yuan, B. (1995). Fuzzy Sets and Fuzzy Logic: Theory and Application. New York: Prentice Hall.

Les A. Piegl, \& Tiller, W. (1995). The NURBS Book. Berlin, Germany: Springer. http://dx.doi.org/10.1007/978-3-642-97385-7

Lodwick, W. (2008). Fuzzy Surfaces in GIS and Geographical Analysis: Theory, Analytical Methods, Algorithms, and Applications. USA: Taylor \& Francis Group.

Mendel, J. M. (2001). Uncertain Rule-Based Fuzzy Logic Systems: Introduction and New Directions. Upper Saddle River, NJ: Prentice Hall PTR.

Nurul Ain, A. K., Abd Fatah, W., Gobithaasan, R. U., \& Rozaimi, Z. (2013). Model of Fuzzy B-Spline Interpolation For Fuzzy Data. Far East Journal of Mathematical Sciences (FJMS), 72(2), 269-280.

Rogers, D. F. (2001). An Introduction to NURBS: With Historical Perspective. USA: Academic Press.

Rozaimi, Z., \& Abd Fatah, W. (2010). Chapter 7: Fuzzy Interpolation of Bezier Curves. Fuzzy: From Theory to Applications (pp. 53-60). UiTM: University Publication Centre (UPENA).

Rozaimi, Z., \& Abd Fatah, W. (2012). Fuzzy B-spline Modeling of Uncertainty Data. Applied Mathematical Sciences, 6(140), 6971-6991.

Rozaimi, Z., Abd. Fatah, W., \& Jamaluddin, M. A. (2010a). Confidence fuzzy interval in verification of offline handwriting signature. European Journal of Scientific Research, 47(3), 455-463.

Rozaimi, Z., Abd. Fatah, W., \& Jamaluddin, M. A. (2010b). Verification of complex fuzzy data of offline handwriting signature. European Journal of Scientific Research, 42(4), 565-575.

Salomon, D. (2006). Curves and Surfaces for Computer Graphics. USA: Springer.

Sarfraz, M., \& Razzak, M. F. A. (2002). An Algorithm for Automatic Capturing of the Font Outlines. Computer 
\& Graphics, 26(5), 795-804. http://dx.doi.org/10.1016/S0097-8493(02)00134-6

Yamaguchi, F. (1988). Curves and Surfaces in Computer Aided Geometric Design. Germany: Springer-Verlag. http://dx.doi.org/10.1007/978-3-642-48952-5

Zadeh, L. (1965). Fuzzy Sets. Information and Control, 8, 338-353. http://dx.doi.org/10.1016/S0019-9958(65)90241-X

Zadeh, L. A. (1975). The concept of a linguistic variable and its application to approximate reasoning-Part I-II-III. Information Science, vol. 8, pp. 199-249, pp. 301-357, vol. 9, pp. 43-93.

Zimmermann, H. J. (1985). Fuzzy Set Theory and Its Applications. USA: Kluwer Academic. 